\title{
Realization of Multimode OAM Beams with Almost the Same Divergence Angles
}

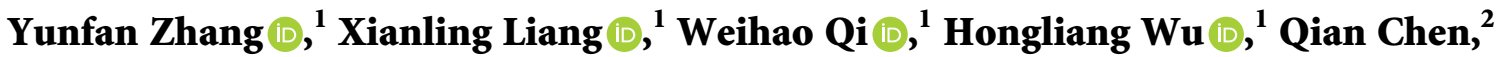 \\ Junping Geng $\mathbb{D}^{1}{ }^{1}$ and Wei Wang ${ }^{3}$
}

${ }^{1}$ Department of Electronics Engineering, Shanghai Jiao Tong University, Shanghai 200240, China
${ }^{2}$ School of Electronics and Information Engineering, Anhui University, Hefei 230039, China
${ }^{3}$ No. 38 Research Institute, China Electronics Technology Group Corporation, Hefei 230088, China

Correspondence should be addressed to Xianling Liang; liangxl@sjtu.edu.cn

Received 27 November 2020; Revised 21 January 2021; Accepted 5 February 2021; Published 18 February 2021

Academic Editor: Mauro Parise

Copyright $(92021$ Yunfan Zhang et al. This is an open access article distributed under the Creative Commons Attribution License, which permits unrestricted use, distribution, and reproduction in any medium, provided the original work is properly cited.

This paper proposes a new method of realizing multimode OAM beams with almost the same divergence angles. The theoretical relationship between the divergence angle of the OAM beam and its radiation source is presented, and the radiation source distributions for various mode OAM beams with the same divergence angle range are discussed. In order to verify this method, an eight-mode OAM antenna constructed by a bifocal parabolic reflector and dual OAM feeds is designed and simulated. The simulation results show that the divergence angle ranges of $3 \mathrm{~dB}$ beamwidth for OAM modes $l= \pm 1, \pm 2, \pm 3, \pm 4$ are $\left(2.2^{\circ}, 4.6^{\circ}\right),\left(2.2^{\circ}, 5.0^{\circ}\right),\left(2.4^{\circ}, 4.4^{\circ}\right)$, and $\left(2.6^{\circ}, 4.8^{\circ}\right)$, the divergence angles corresponding to the maximum beam directions are $3.5^{\circ}, 3.6^{\circ}, 3.5^{\circ}$, and $3.8^{\circ}$, respectively, and the maximum difference is within $0.3^{\circ}$.

\section{Introduction}

Since Allen et al. first discovered OAM (orbital angular momentum) in 1992 [1] and Thide et al. verified the multiplexing transmission capability of OAM electromagnetic waves in the radio frequency band in 2007 [2], the research on OAM antennas has attracted widespread attention [3-5]. From the published literature, many methods were proposed to generate OAM waves at radio frequency, such as crafted antennas [6], loop antennas [7], OAM phase plates [8], and circular antenna arrays $[9,10]$. It is worth noting that the essential feature of OAM antennas is the beam divergence, which results in the phase space distribution expansion with the increase of distance and makes it difficult to receive and demodulation. Literature studies $[11,12]$ introduced lens and reflectors to decrease the beam divergence angles of OAM antennas, but the beam divergence angles of various OAM modes are different. To achieve the same divergence angle for different OAM modes, Zhang et al. proposed an OAM antenna based on a ring slit resonator, which can generate omnidirectional OAM beams [13], this method effectively realizes the consistency of beam divergence angles for various OAM modes, but the beam divergence angle is $90^{\circ}$ due to its omnidirectional radiation. Qin et al. proposed a four-mode UCA (uniform circular array) antenna design [14], through optimization of the element positions, and the beam with OAM modes of $l=-1,-2$, and -3 and the divergence angle of $18^{\circ}$ was generated simultaneously.

In short, there is little literature discussing in principle how to control the divergence angles of multimode OAM beams. Different from the work mentioned [13, 14], in this paper, the theoretical relationship between the divergence angle of the OAM beam and its radiation source is presented, and the radiation source distributions for various mode OAM beams with the same divergence angle range are discussed. Based on this, a bifocal parabolic reflector antenna with dual OAM feeds is proposed as an example to achieve the corresponding equivalent source distributions and verify the design of eight-mode OAM beams with almost the same divergence angles. 


\section{Theory and Analysis}

Figure 1 shows an OAM radiation source distribution, where the current loop is with radius $R$ and current density $J_{R}$. It is assumed that the amplitude of the current density $J_{R}$ in the loop is uniform, and the phase is periodically distributed with $e^{j l \varphi}$, where $l$ is the OAM mode. The vector magnetic potential of its radiation is expressed as follows:

$$
\begin{aligned}
\vec{A}(r) \approx & \vec{a}_{\varphi} \frac{(-j)^{l+1} \mu_{0} R J_{R} e^{j k r} e^{j l \varphi}}{4 \pi r} \\
& \cdot\left[J_{|l|+1}(k R \sin \theta)-J_{|l|-1}(k R \sin \theta)\right], \quad l= \pm 1, \pm 2 \ldots
\end{aligned}
$$

According to the far-field approximation conditions [15], the corresponding electric field can be approximately expressed as

$$
\begin{array}{r}
\vec{E} \approx \vec{a}_{\varphi} \frac{(-j)^{l} \omega \mu_{0} R J_{R} e^{j k r} e^{j l \varphi}}{4 \pi r} \cdot\left[J_{|l|+1}(k R \sin \theta)-J_{|l|-1}(k R \sin \theta)\right], \\
l= \pm 1, \pm 2 \ldots
\end{array}
$$

To obtain the maximum $\vec{E}$, we can get

$$
\left[J_{|l|+1}(k R \sin \theta)-J_{|l|-1}(k R \sin \theta)\right]^{\prime}=0, \quad l= \pm 1, \pm 2 \ldots
$$

According to the property of Bessel function, the above formula can be simplified to

$$
J_{|l|}^{\prime \prime}(k R \sin \theta)=0, \quad l= \pm 1, \pm 2 \ldots
$$

The value of $k R \sin \theta$ is the eigenvalue of the secondorder Bessel function, $k$ is the wave number, and $\theta$ is the beam divergence angle. Table 1 shows eigenvalues of the second-order Bessel function for $|l|=1,2,3$, and 4 . Firstly, from the expression of $k R \sin \theta$, it is noted that the larger $R$, the smaller $\theta$. Secondly, the values of $k R \sin \theta$ are different for various $|l|$; the higher order $|l|$, the larger $\theta$, as shown in Figure 2.

Assuming a target OAM beam, its angle range of $3 \mathrm{~dB}$ beamwidth is $\theta \in\left(3^{\circ}, 4^{\circ}\right)$ and the corresponding divergence angle range is also $\theta \in\left(3^{\circ}, 4^{\circ}\right)$. Then, the radiation source distributions for various OAM modes can be obtained, $R_{1} \in(3.5,4.7) \quad \lambda_{0}, R_{2} \in(6.0,8.0) \quad \lambda_{0}, R_{3} \in(8.4,11.2) \lambda_{0}$, and $R_{4} \in(10.7,14.3) \lambda_{0}$, as shown in Figure 3 , where $\lambda_{0}$ is the operation wavelength in free space. This means that the beams of these modes will have the same divergence angle range as long as each mode is distributed according to the above radiation source.

In order to achieve the abovementioned OAM source distributions of modes $|l|=1,2,3,4$, we propose a bifocal parabolic reflector with dual OAM feeds, as shown in Figure 4. The reflector is composed of two paraboloids with different focal lengths $\left(f_{1}, f_{2}\right)$ and aperture sizes $\left(D_{1}, D_{2}\right)$, which are denoted by part $\mathrm{A}$ and part $\mathrm{B}$. The geometric expressions of the two paraboloids are as follows:

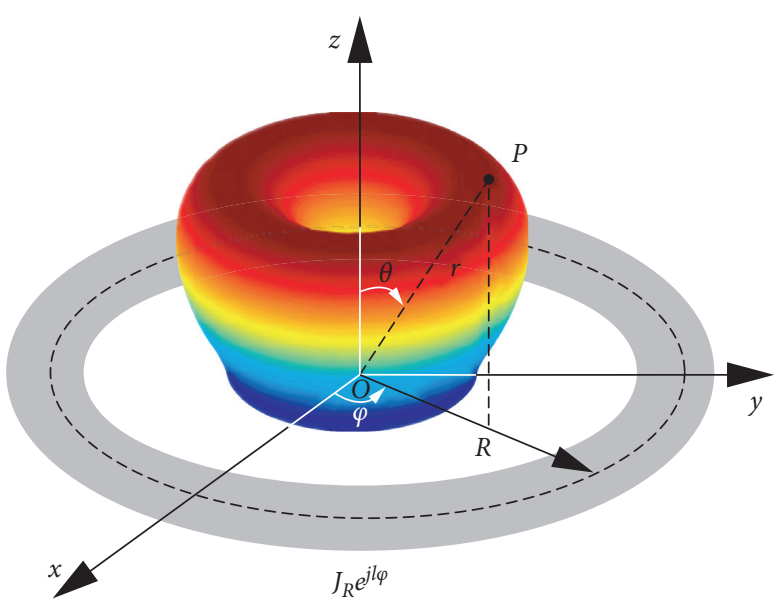

Figure 1: OAM radiation source.

TABLE 1: Eigenvalues of second-order Bessel's equation.

\begin{tabular}{lcccc}
\hline$|l|$ & 1 & 2 & 3 & 4 \\
\hline$k R \sin \theta$ & 1.55 & 2.63 & 3.68 & 4.70 \\
\hline
\end{tabular}

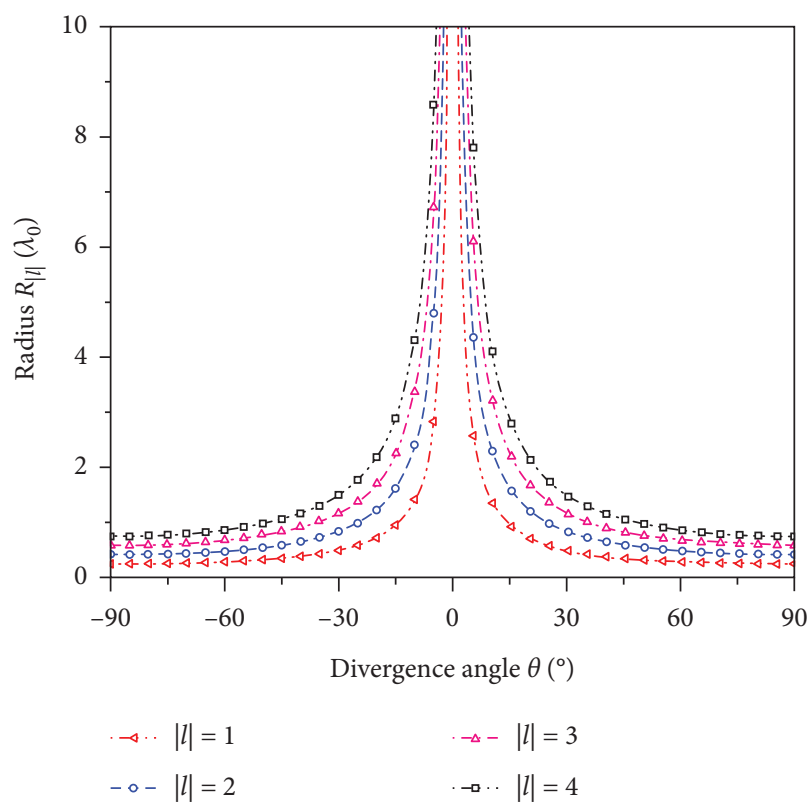

Figure 2: Radius of source vs. divergence angle.

$$
\left\{\begin{array}{l}
x^{2}+y^{2}=4 f_{1}(z+\Delta z), \\
\sqrt{x^{2}+y^{2}} \in\left(R_{1 \min }, R_{2 \max }\right), \\
x^{2}+y^{2}=4 f_{2} z, \\
\sqrt{x^{2}+y^{2}} \in\left(R_{3 \min }, R_{4 \max }\right),
\end{array}\right.
$$

where $f_{1}$ is the focal length of part A and OAM feed 1 is located at this focal position. $f_{2}$ is the focal length of part $\mathrm{B}$ and OAM feed 2 is located at that focal position.

The equivalent radiation source distributions of the proposed bifocal parabolic reflector antenna can be 


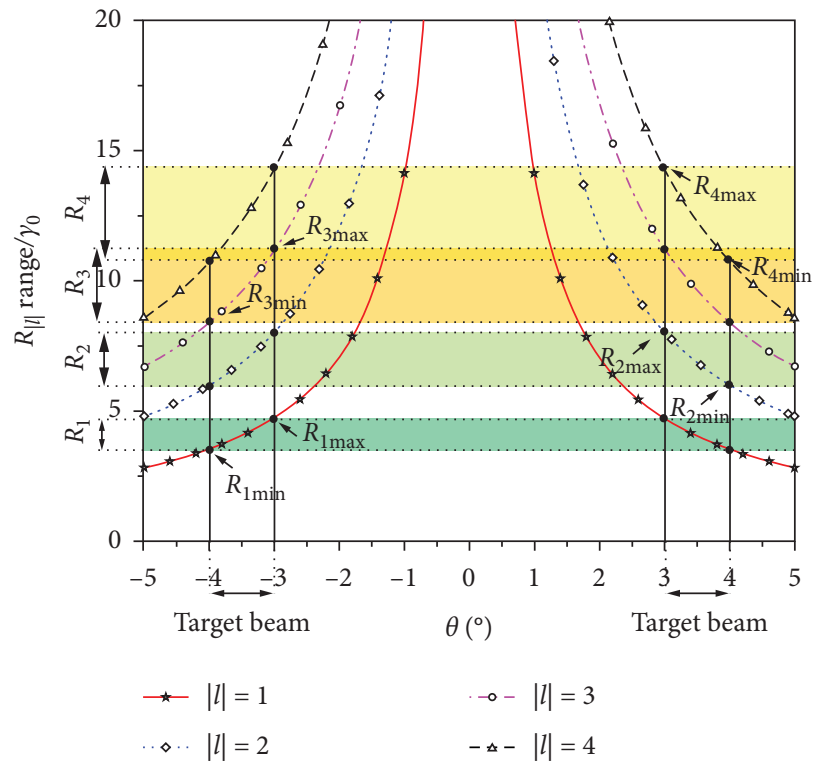

FIgURE 3: Source distributions of various OAM modes.

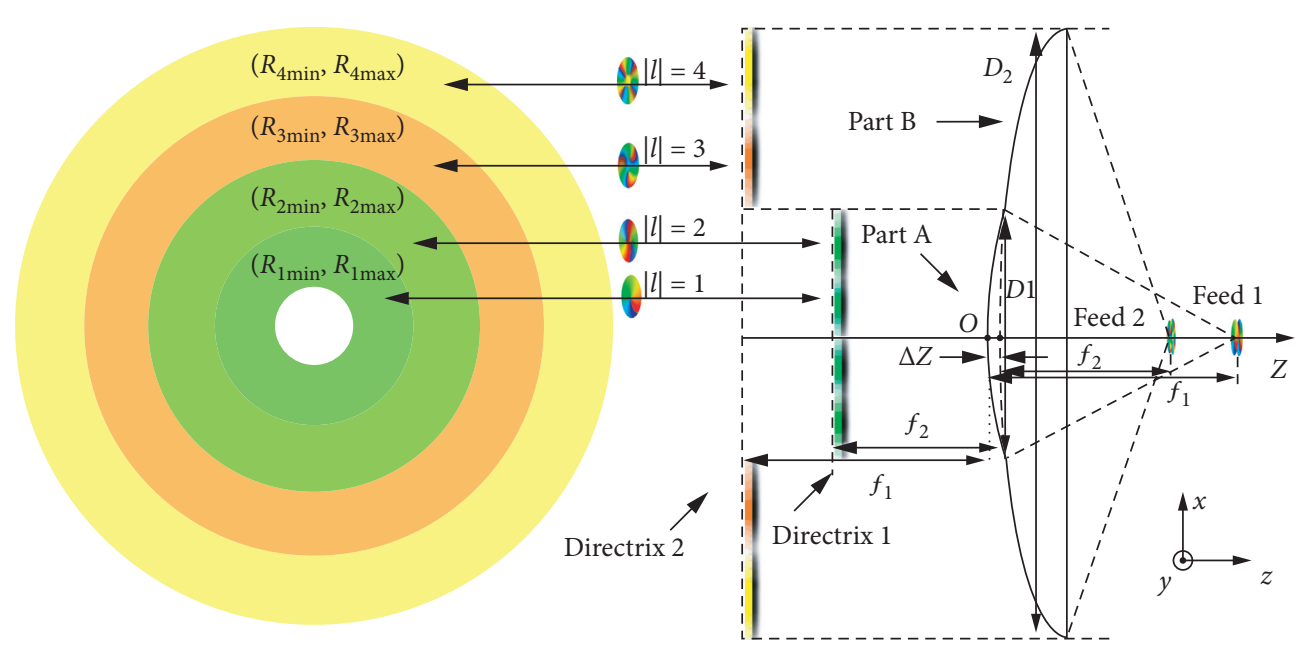

Figure 4: Schematic diagram of bifocal parabolic reflector antenna.

explained as follows. OAM feed 1 is used to generate OAM beams of $|l|=1$ and 2, and OAM feed 2 is used to generate OAM beams of $|l|=3$ and 4 . According to the nature of the parabolic reflector antenna, the radiation formed by feed 1 beams incident on the reflecting surface part A can be regarded as the equivalent source radiation at the position of directrix 1. The radiation formed by feed 2 beams incident on the reflecting surface part B can be regarded as the equivalent source radiation at the position of directrix 2. Therefore, the source distribution required by the above modes $|l|=1,2,3,4$ can be formed by optimizing sizes of the two paraboloids and beamwidth of dual OAM feeds. According to Figure 3, the aperture sizes of two paraboloids need to meet the conditions: $R_{3 \min } \geq\left(D_{1} / 2\right) \geq R_{2 \max }$ and $\left(D_{2} / 2\right) \geq R_{4 \max }$, where $D_{1}$ is the diameter of part $\mathrm{A}$ and $D_{2}$ is the diameter of part $\mathrm{B}$.
Then, the OAM feed beams can be generated from the geometric relationship between the parabolic reflector and the dual OAM feeds, as follows:

$$
\begin{cases}\theta_{|l|}^{\prime}=2 \operatorname{arccot}\left(\frac{4 f_{1}}{R_{|l|}}\right), & |l|=1,2, \\ \theta_{|l|}^{\prime}=2 \operatorname{arccot}\left(\frac{4 f_{2}}{R_{|l|}}\right), & |l|=3,4 .\end{cases}
$$

We choose $D_{1}=16.8 \lambda_{0}, D_{2}=28.6 \lambda_{0}, f_{1}=12.6 \lambda_{0}$, and $f_{2}=10.3 \lambda_{0}$ based on the above analysis. The feed beam range corresponding to source distribution of each mode can be calculated according to formula (6): $\theta_{1}^{\prime} \in\left(16.0^{\circ}\right.$, $\left.21.2^{\circ}\right), \theta_{2}^{\prime} \in\left(26.8^{\circ}, 35.2^{\circ}\right), \theta_{3}^{\prime} \in\left(44.4^{\circ}, 57.1^{\circ}\right)$, and $\theta_{4}^{\prime} \in\left(55.0^{\circ}\right.$, $\left.69.6^{\circ}\right)$, as shown in Table 2 . 
TABLE 2: Feed beams for various OAM modes.

\begin{tabular}{lccr}
\hline$l$ & Target beam range $\theta$ & Source distribution $R_{l}$ & Feed beam range $\theta_{l}^{\prime}$ \\
\hline 1 & & $(3.5,4.7) \lambda_{0}$ & $\left(16.0^{\circ}, 21.2^{\circ}\right)$ \\
2 & $\left(3^{\circ}, 4^{\circ}\right)$ & $(6.0,8.0) \lambda_{0}$ & $\left(26.8^{\circ}, 35.2^{\circ}\right)$ \\
3 & & $(8.4,11.2) \lambda_{0}$ & $\left(44.4^{\circ}, 57.1^{\circ}\right)$ \\
4 & & $(10.7,14.3) \lambda_{0}$ & $\left(55.0^{\circ}, 69.6^{\circ}\right)$ \\
\hline
\end{tabular}

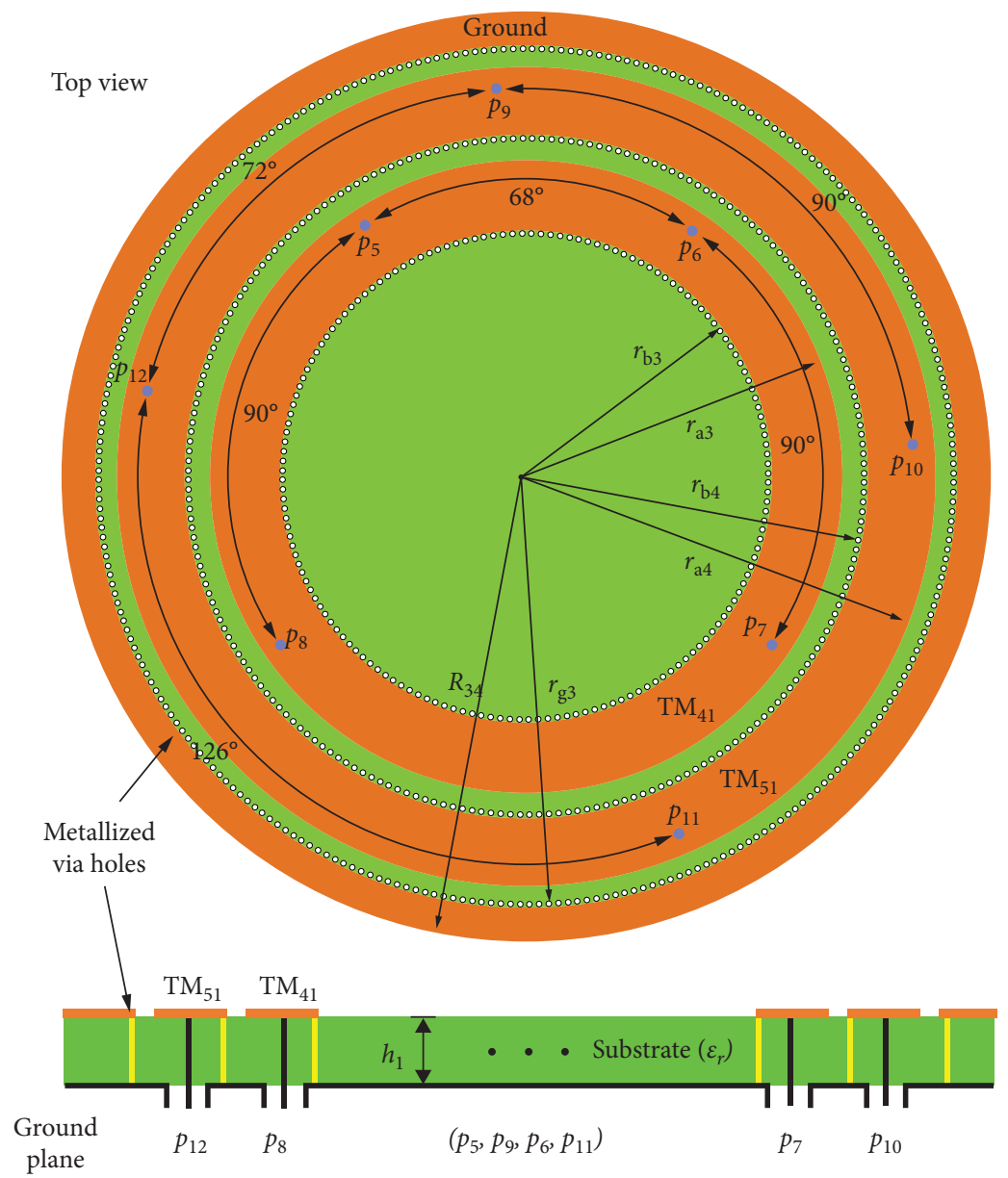

Figure 5: Structure of feed 2.

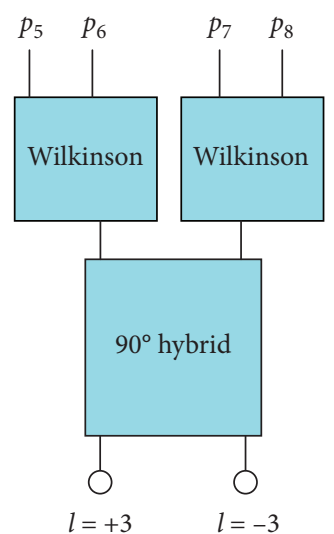

Feeding network $(l= \pm 3)$

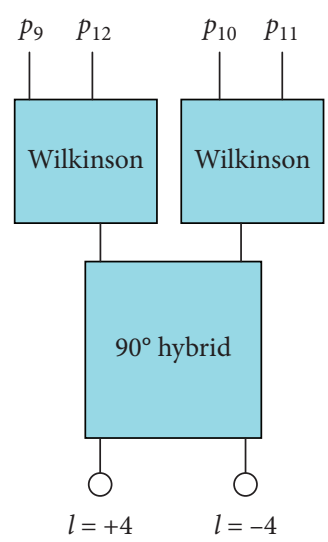

Feeding network $(l= \pm 4)$

TABLE 3: Excitation amplitude and phase of various modes.

\begin{tabular}{lccr}
\hline$l$ & Feeding ports & Amplitude & Phase $\left(^{\circ}\right)$ \\
\hline+1 & $p_{1}, p_{2}$ & 1,1 & 900 \\
-1 & $p_{1}, p_{2}$ & 1,1 & 090 \\
+2 & $p_{3}, p_{4}$ & 1,1 & 900 \\
-2 & $p_{3}, p_{4}$ & 1,1 & 090 \\
+3 & $p_{5}, p_{6}, p_{7}, p_{8}$ & $1,1,1,1$ & 909000 \\
-3 & $p_{5}, p_{6}, p_{7}, p_{8}$ & $1,1,1,1$ & 009090 \\
+4 & $p_{9}, p_{10}, p_{11}, p_{12}$ & $1,1,1,1$ & 900090 \\
-4 & $p_{9}, p_{10}, p_{11}, p_{12}$ & $1,1,1,1$ & 090900 \\
\hline
\end{tabular}




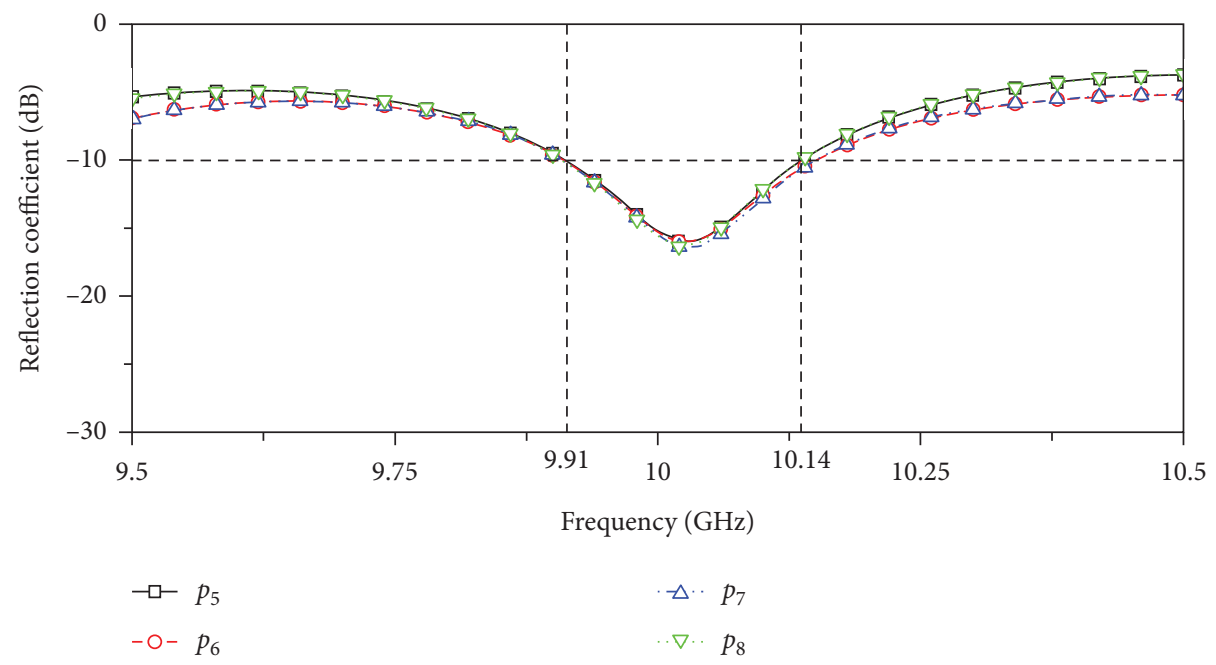

(a)

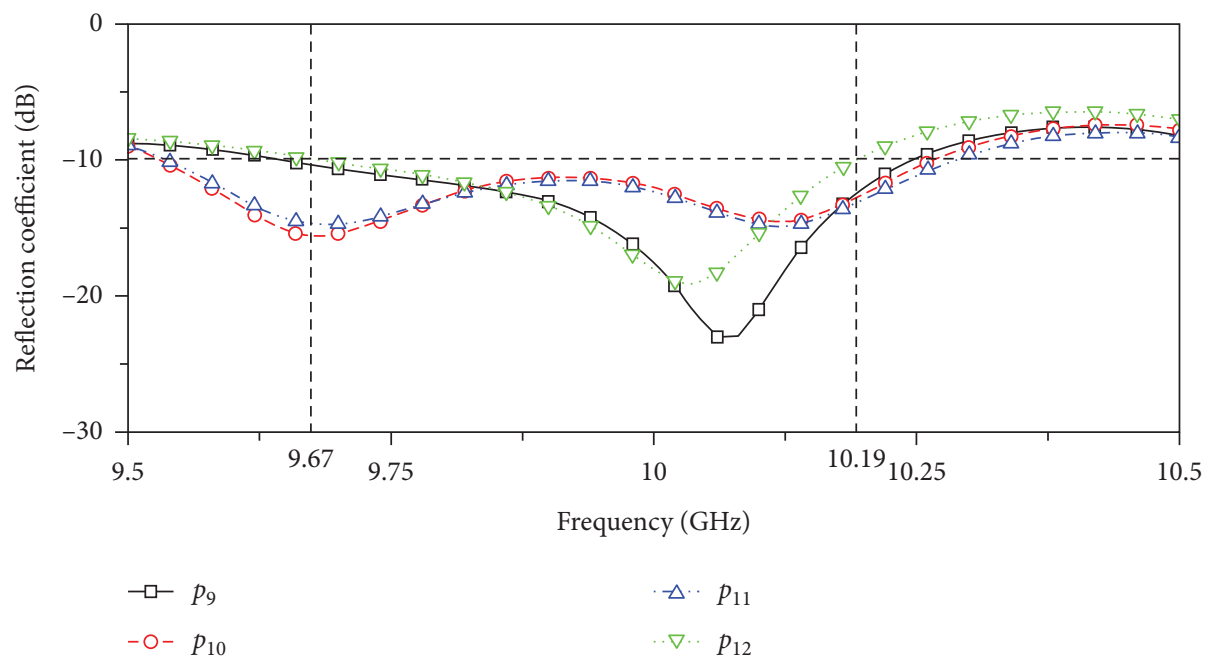

(b)

Figure 6: Simulated reflection coefficients of feed 2. (a) $l= \pm 3$. (b) $l= \pm 4$.

\section{Simulation and Discussion}

If a circular microstrip antenna works in $\mathrm{TM}_{(|l|+1) 1}$ mode and is excited by two feeding points with a certain phase, it can produce OAM beam with the mode of $|l|[16,17]$. In order to achieve the mentioned feed beams, feed 2 adopts a dual-ring microstrip antenna $[18,19]$, as shown in Figure 5.

The inner side of each ring is grounded with a set of metallized via holes, which is equivalent to a shorting wall, and the outer side is equivalent to a magnetic current loop as the radiation source [20]. Each ring is simultaneously excited by four feeding ports separated by a certain angle, where $p_{5}$ to $p_{8}$ are used for exciting the inner ring patch and $p_{9}$ to $p_{12}$ are used for exciting the outer ring patch. The excitation amplitude and phase are shown in Table 3, which can be achieved by two feeding networks with four Wilkinson dividers and two $90^{\circ}$ hybrids. The inner ring patch works in the $\mathrm{TM}_{41}$ mode to generate OAM beams of $l= \pm 3$, and the outer ring patch works in the $\mathrm{TM}_{51}$ mode to generate OAM beams of $l= \pm 4$. Particularly, an additional grounded ring is used to reduce the back radiation. Based on the full-wave HFSS simulation, the optimized parameters are as follows: $r_{b 3}=15.3 \mathrm{~mm}, r_{a 3}=19.5 \mathrm{~mm}, r_{b 4}=21.1 \mathrm{~mm}, r_{a 4}=24.9 \mathrm{~mm}$, $r_{g 3}=26.5 \mathrm{~mm}, R_{34}=30 \mathrm{~mm}, \epsilon_{r}=3.66, h_{1}=0.762 \mathrm{~mm}$, and the diameter of the via hole is $0.2 \mathrm{~mm}$ and the spacing is $1.1 \mathrm{~mm}$. The simulated reflection coefficients of all ports are shown in Figure 6. 

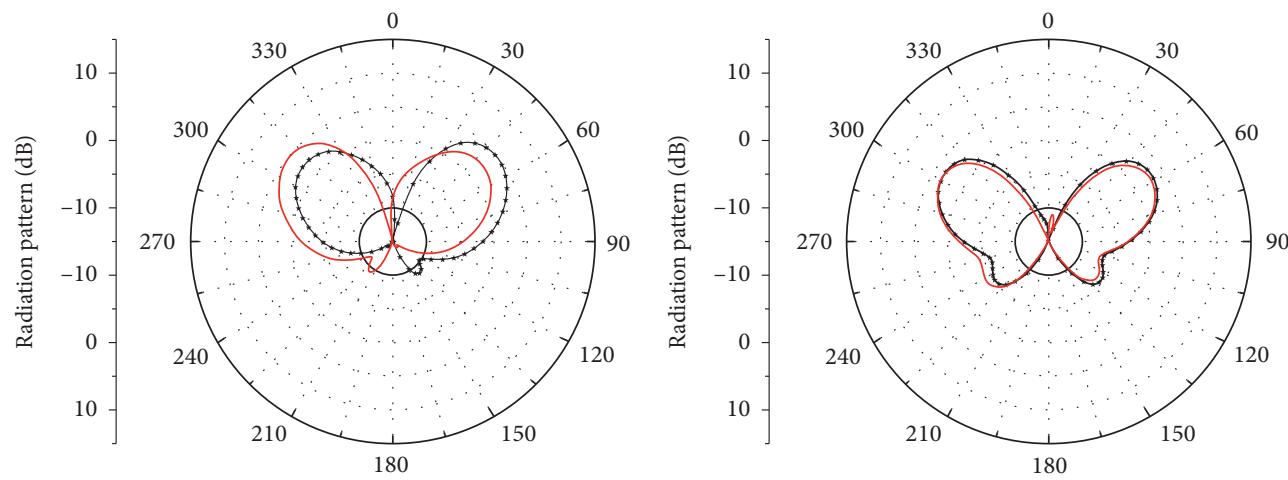

$l=+3$
$-l=-3$

$-l=+4$
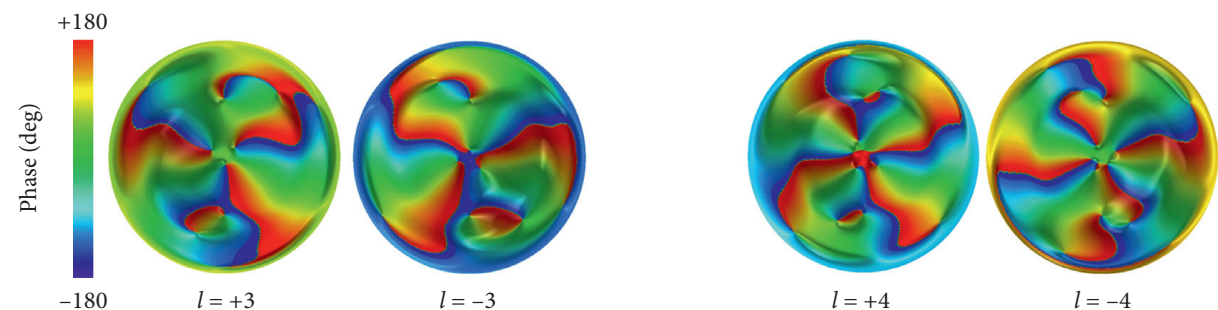

Figure 7: Radiation patterns of feed 2.
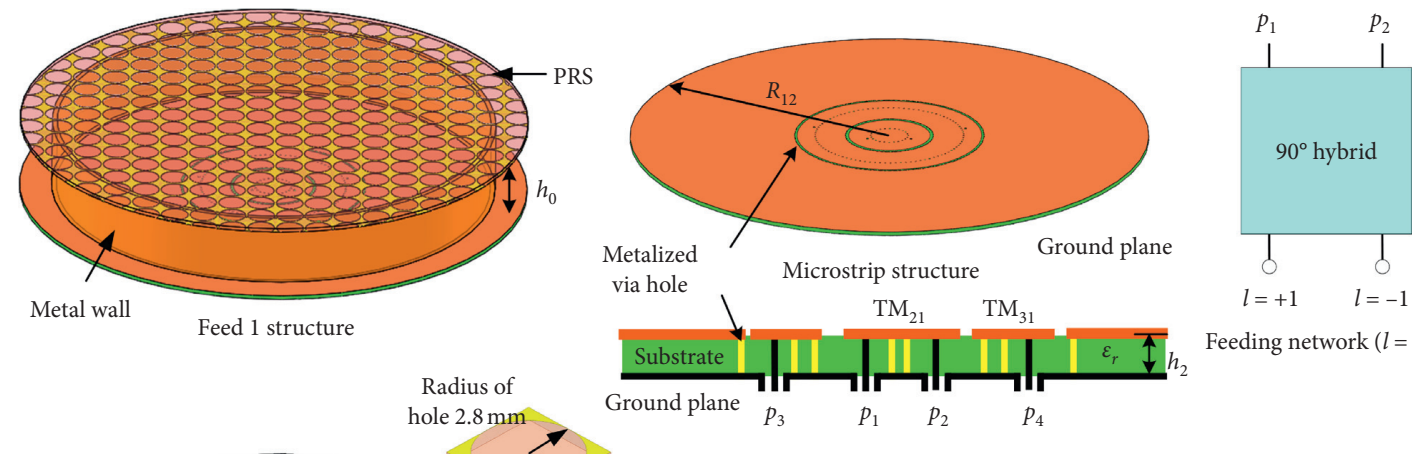

Feeding network $(l= \pm 1)$

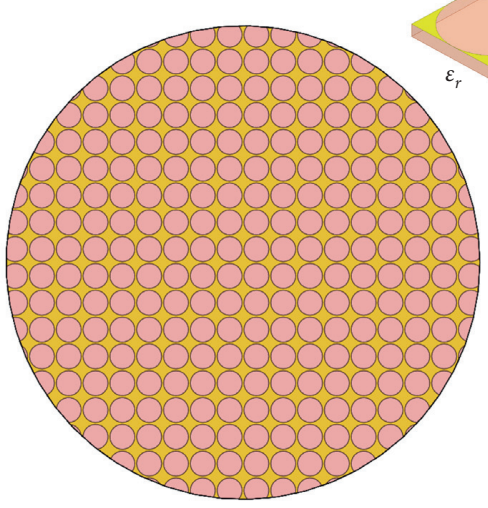

PRS
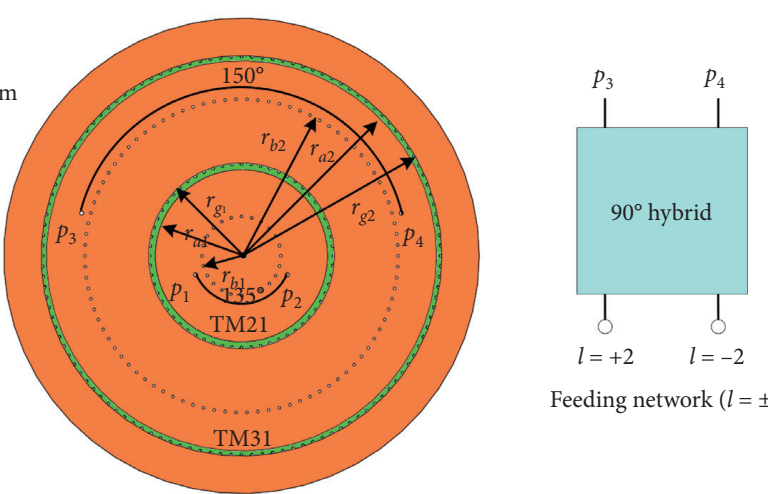

Feeding network $(l= \pm 2)$

FIgURE 8: Structure of feed 1. 


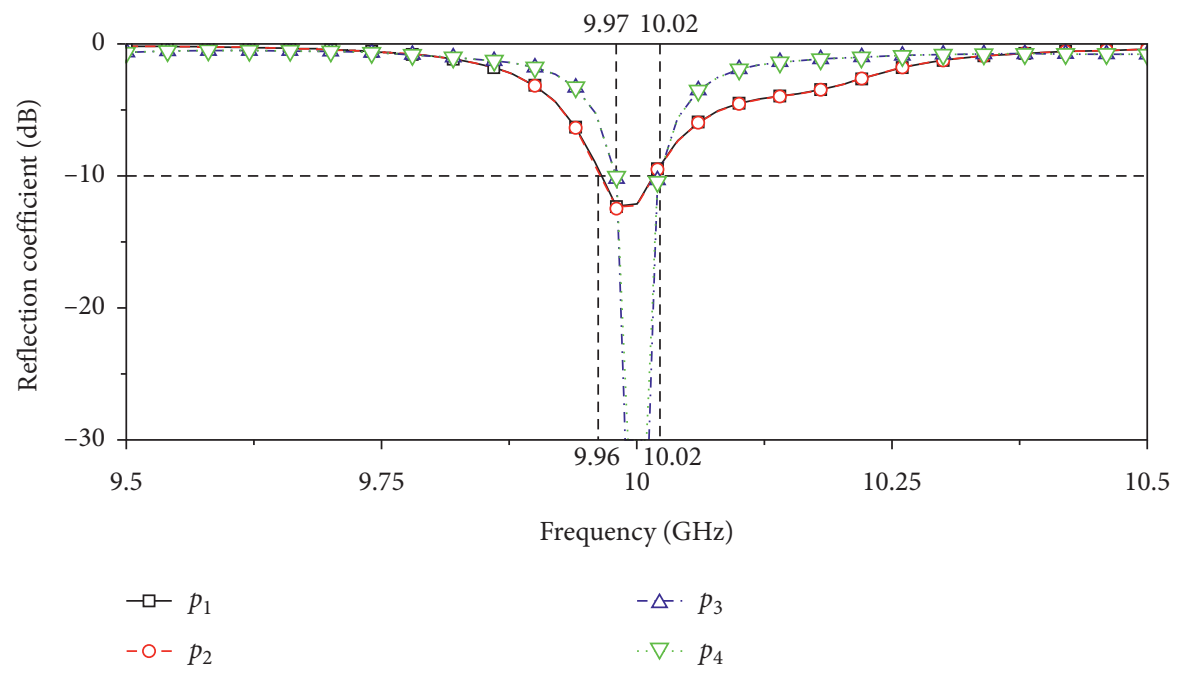

FIGURE 9: Simulated reflection coefficients of feed 1.
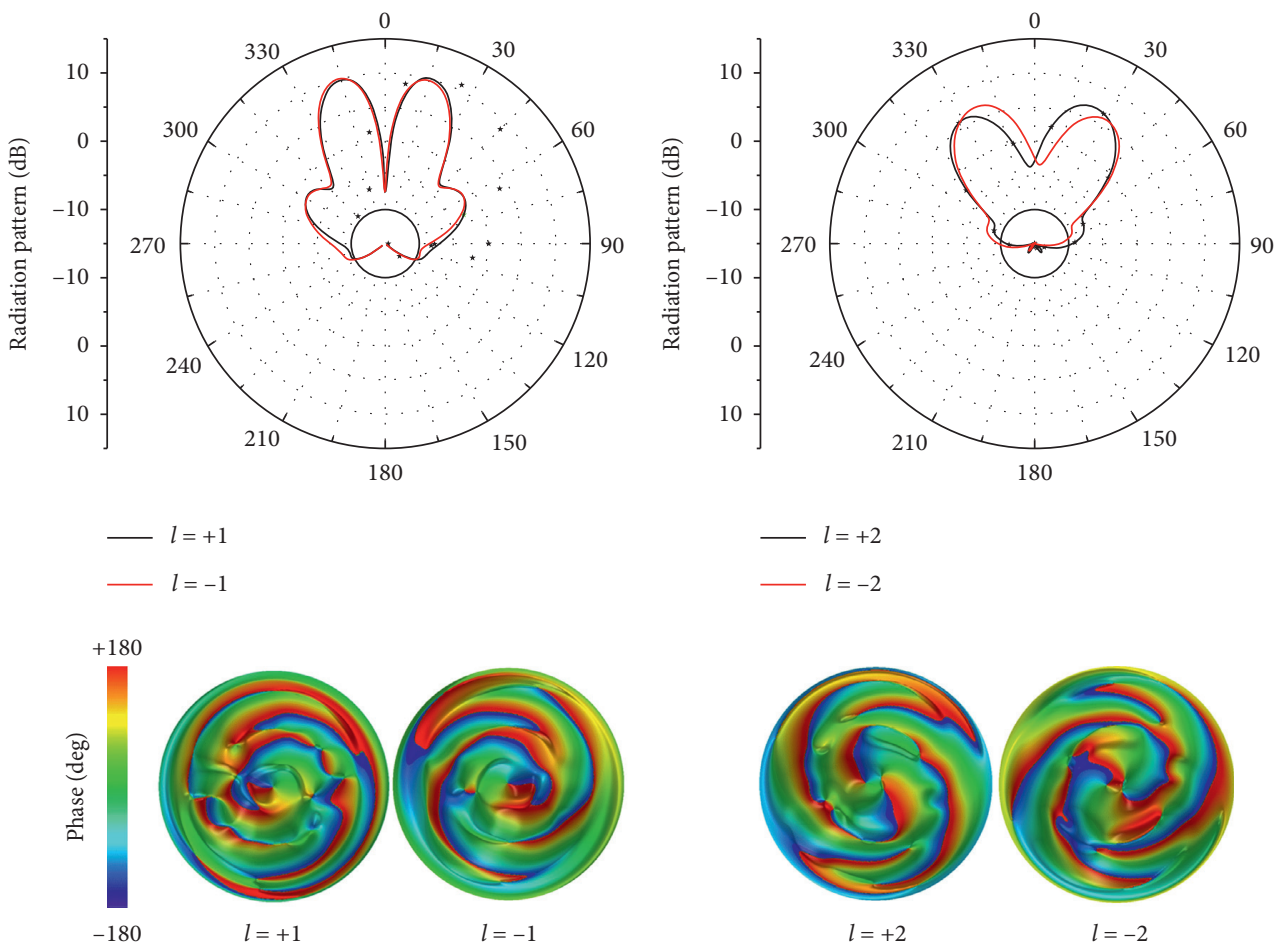

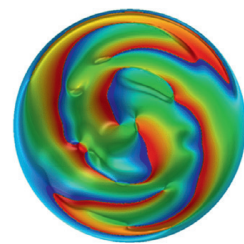

$l=+2$

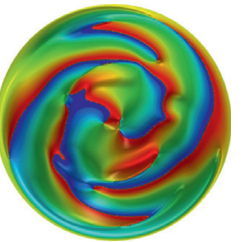

$l=-2$

Figure 10: Radiation patterns of feed 1.

The $10 \mathrm{~dB}$ impedance bandwidth of mode $l= \pm 3$ is from $9.91 \mathrm{GHz}$ to $10.14 \mathrm{GHz}$, and that of mode $l= \pm 4$ is from $9.67 \mathrm{GHz}$ to $10.19 \mathrm{GHz}$. The corresponding radiation patterns of feed 2 are shown in Figure 7, which can cover the feed 1 beam requirements of $\theta_{ \pm 3}^{\prime}$ and $\theta_{ \pm 4}^{\prime}$, but wider than required. The main reason is that the OAM beam divergence is related to its beamwidth, and it is not easy to change the beamwidth under the fixed beam direction.

Considering that the beam divergence angle range of feed 1 is much smaller than that of feed 2, it is hard to satisfy the feed beam requirements by using the microstrip antenna alone. Therefore, the combination of the microstrip antenna and the Fabry-Perot cavity is used [21], as shown in Figure 8.

Each patch is simultaneously excited by two feeding ports separated by a certain angle, where $p_{1}$ and $p_{2}$ are used for exciting the inner circular patch and $p_{3}$ and $p_{4}$ are used for exciting the outer ring patch. Similar to feed 2, the inner circular patch works in the $\mathrm{TM}_{21}$ mode to generate OAM beams of $l= \pm 1$ and the outer ring patch works in the $\mathrm{TM}_{31}$ mode to generate OAM beams of $l= \pm 2$. The excitation 


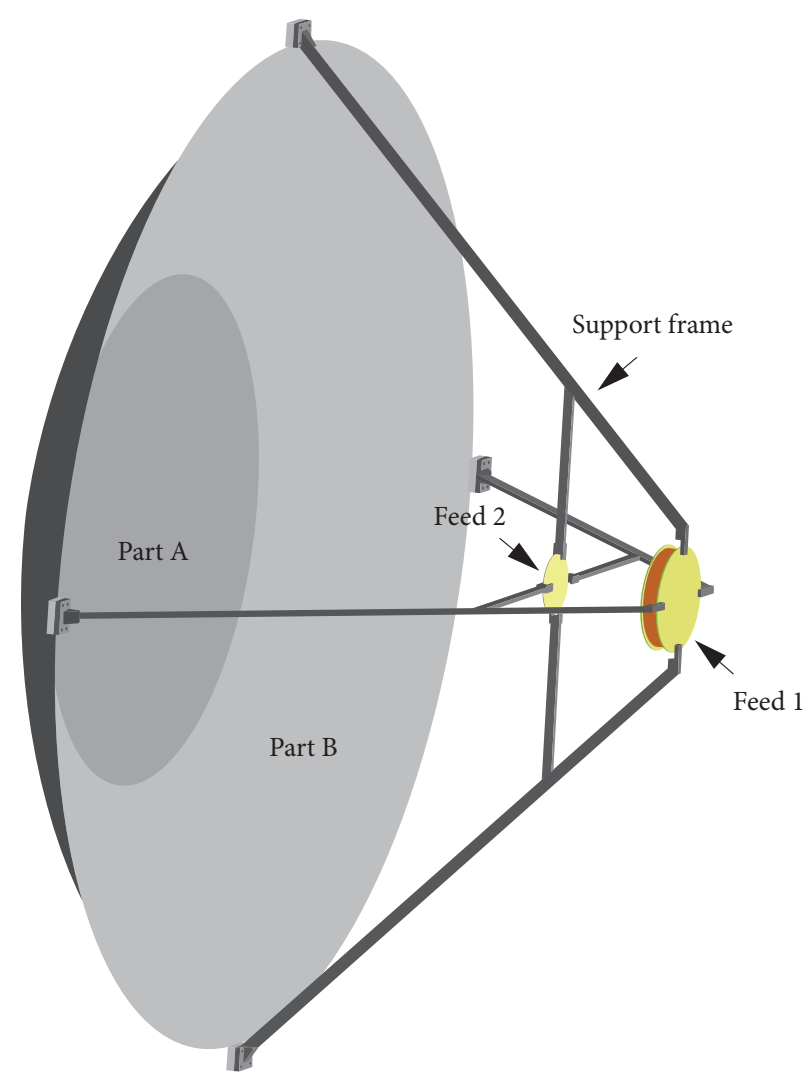

FiguRE 11: Configuration of bifocal reflector antenna.

amplitude and phase are also shown in Table 3, which can be achieved by two $90^{\circ}$ hybrids. The principle is that the microstrip antenna radiates OAM electromagnetic waves, some of them are directly transmitted from the partially reflective surface (PRS), while others are reflected. When the reflected OAM waves reach the ground and the metal wall, they will be reflected to the PRS with the same phase. After multiple reflections and transmissions, the radius of the radiation source increases to a certain extent, thereby narrowing the beam divergence angle range. The optimized parameters are as follows: $r_{b 1}=3.8 \mathrm{~mm}, r_{a 1}=8.3 \mathrm{~mm}$, $r_{b 2}=15.2 \mathrm{~mm}, r_{a 2}=18.9 \mathrm{~mm}, r_{g 1}=9 \mathrm{~mm}, r_{g 2}=19.4 \mathrm{~mm}$, $\epsilon_{r}=3.66, h_{2}=0.762 \mathrm{~mm}, h_{0}=14.7 \mathrm{~mm}$, the diameter of the via hole is $0.2 \mathrm{~mm}$, and the spacing is $0.95 \mathrm{~mm}$. The simulated reflection coefficients of all ports are shown in Figure 9.

The $10 \mathrm{~dB}$ impedance bandwidth of mode $l= \pm 1$ is from $9.96 \mathrm{GHz}$ to $10.02 \mathrm{GHz}$, and that of mode $l= \pm 2$ is from $9.97 \mathrm{GHz}$ to $10.02 \mathrm{GHz}$. It is noted that the impedance bandwidth of feed 1 is much smaller than that of feed 2 . The main reason is the Fabry-Perot cavity limits the impedance bandwidth due to the phase synchronization of the OAM wave multiple reflections. The radiation patterns of feed 1 are shown in Figure 10, which can cover feed 1 beam requirements of $\theta_{ \pm 1}^{\prime}$ and $\theta_{ \pm 2}^{\prime}$.
Based on the above dual OAM feeds, the bifocal parabolic reflector antenna is designed. Its optimized parameters are $D_{1}=480 \mathrm{~mm}, D_{2}=816 \mathrm{~mm}, f_{1}=360 \mathrm{~mm}$, and $f_{2}=294 \mathrm{~mm}$. The configuration of the bifocal parabolic reflector antenna is shown in Figure 11. The dual OAM feeds and the bifocal reflector are fixed by a nylon support frame. Figure 12 shows the simulated radiation patterns of various OAM modes. The divergence angle ranges of $3 \mathrm{~dB}$ beamwidth for OAM modes $l= \pm 1, \pm 2, \pm 3, \pm 4$ are $\left(2.2^{\circ}, 4.6^{\circ}\right),\left(2.2^{\circ}, 5.0^{\circ}\right),\left(2.4^{\circ}, 4.4^{\circ}\right)$, and $\left(2.6^{\circ}, 4.8^{\circ}\right)$, respectively, which are slightly wider than the target's $3 \mathrm{~dB}$ OAM beamwidth. The main reason is that the beamwidth of feed 1 and feed 2 is wider than required. The divergence angles corresponding to the maximum beam directions for OAM modes $l= \pm 1, \pm 2, \pm 3, \pm 4$ are $3.5^{\circ}, 3.6^{\circ}, 3.5^{\circ}, 3.8^{\circ}$, respectively, and the maximum difference is within $0.3^{\circ}$, which means that eight-mode OAM beams almost have the same divergence angles. Moreover, the gains and side lobe levels for OAM modes $l= \pm 1, \pm 2, \pm 3, \pm 4$ are $28.2 \mathrm{dBi}, 27.6 \mathrm{dBi}, 28.0 \mathrm{dBi}$, and $26.0 \mathrm{dBi}$, and $-14.1 \mathrm{~dB},-11.1 \mathrm{~dB},-12.0 \mathrm{~dB}$, and $-7.6 \mathrm{~dB}$, respectively. Compared with literature $[13,14]$, the proposed antenna has more OAM modes, smaller divergence angles, and lower side lobe levels. 

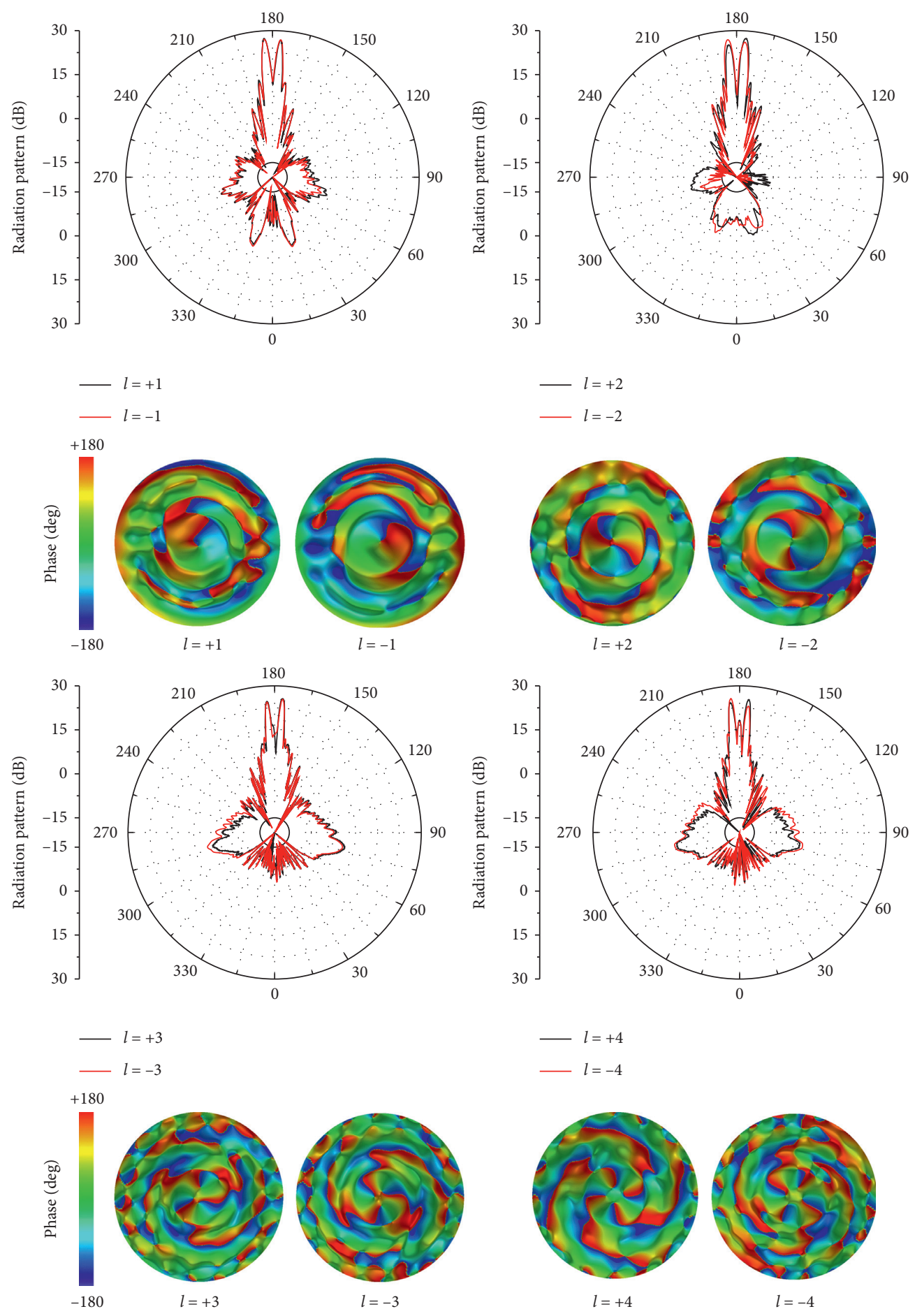

FIGURE 12: Radiation patterns of bifocal parabolic reflector antenna.

\section{Conclusion}

A new method of realizing multimode OAM beams with almost the same divergence angles has been presented in this paper. By establishing the relationship between the divergence angle of the OAM beam and its radiation source, the radiation source distributions of various mode OAM beams for the same divergence angle range are discussed. To verify this method, an eight-mode OAM antenna constructed by a bifocal parabolic reflector and dual OAM feeds has also been proposed, designed, and simulated. The simulation results show that the divergence angle ranges of $3 \mathrm{~dB}$ beamwidth for OAM modes $l= \pm 1, \pm 2, \pm 3, \pm 4 \quad$ are $\quad\left(2.2^{\circ}, 4.6^{\circ}\right),\left(2.2^{\circ}, 5.0^{\circ}\right),\left(2.4^{\circ}\right.$, $\left.4.4^{\circ}\right)$, and $\left(2.6^{\circ}, 4.8^{\circ}\right)$, the divergence angles corresponding to the maximum beam directions are $3.5^{\circ}, 3.6^{\circ}, 3.5^{\circ}$, and $3.8^{\circ}$, respectively, and the maximum difference is within $0.3^{\circ}$. This method is also suitable for other forms of multimode OAM antennas to achieve the same divergence angles. 


\section{Data Availability}

The data used to support the findings of this study are included within the article.

\section{Conflicts of Interest}

The authors declare that they have no conflicts of interest.

\section{Acknowledgments}

This work was supported by the National Natural Science Foundation of China under Grant 61801447.

\section{References}

[1] L. Allen, M. W. Beijersbergen, R. J. C. Spreeuw, and J. P. Woerdman, "Orbital angular momentum of light and the transformation of laguerre-Gaussian laser modes," Physical Review A, vol. 45, no. 11, pp. 8185-8189, 1992.

[2] B. Thide, H. Then, J. Sjoholm et al., "Utilization of photon orbital angular momentum in the low-frequency radio domain," Physical Review Letters, vol. 99, no. 8, p. 87701, 2007.

[3] Y. Yao, X. Liang, M. Zhu, W. Zhu, J. Geng, and R. Jin, "Analysis and experiments on reflection and refraction of orbital angular momentum waves," IEEE Transactions on Antennas and Propagation, vol. 67, no. 4, pp. 2085-2094, 2019.

[4] Y. Yan, G. Xie, M. P. J. Lavery et al., "High-capacity millimetre-wave communications with orbital angular momentum multiplexing," Nature Communications, vol. 5, p. 4876, 2014.

[5] Y. Yao, X. Liang, W. Zhu, J. Geng, and R. Jin, "Experiments of orbital angular momentum phase properties for long-distance transmission," IEEE Access, vol. 7, pp. 62689-62694, 2019.

[6] H.-H. Lv, Q.-L. Huang, X.-J. Yi, J.-Q. Hou, and X.-W. Shi, "Low-profile transmitting metasurface using single dielectric substrate for OAM generation," IEEE Antennas and Wireless Propagation Letters, vol. 19, no. 5, pp. 881-885, 2020.

[7] L. Yu, X. Li, Z. Qi, H. Zhu, Y. Huang, and Z. Akram, "Wideband circularly polarized dual-mode vortex beams reflectarray design using dual-semi-split-loop elements," IEEE Antennas and Wireless Propagation Letters, vol. 18, no. 12, pp. 2676-2680, 2019.

[8] X. Hui, S. Zheng, Y. Hu et al., "Ultralow reflectivity spiral phase plate for generation of millimeter-wave OAM beam," IEEE Antennas and Wireless Propagation Letters, vol. 14, pp. 966-969, 2015.

[9] K. Liu, Y. Cheng, Z. Yang, H. Wang, Y. Qin, and X. Li, "Orbital-angular-momentum-based electromagnetic vortex imaging," IEEE Antennas and Wireless Propagation Letters, vol. 14, pp. 711-714, 2015.

[10] Y. Yao, X. Liang, W. Zhu, J. Geng, and R. Jin, "Phase mode analysis of radio beams carrying orbital angular momentum," IEEE Antennas and Wireless Propagation Letters, vol. 16, pp. 1127-1130, 2017.

[11] X. Bai, F. Kong, J. Qian et al., "Polarization-insensitive metasurface lens for efficient generation of convergent OAM beams," IEEE Antennas and Wireless Propagation Letters, vol. 18, no. 12, pp. 2696-2700, 2019.

[12] X. Bai, A. Cao, F. Kong et al., "Rotman lens-fed fabry-perot resonator antennas for generating converged multi-mode OAM beams," IEEE Access, vol. 7, pp. 105768-105775, 2019.

[13] Z. Zhang, S. Zheng, X. Jin, H. Chi, and X. Zhang, "Generation of plane spiral OAM waves using traveling-wave circular slot antenna," IEEE Antennas Wirel. Propag. Lett. vol. 16, pp. 8-11, 2017.

[14] F. Qin, L. Li, Y. Liu, W. Cheng, and H. Zhang, "A four-mode OAM antenna array with equal divergence angle," IEEE Antennas and Wireless Propagation Letters, vol. 18, no. 9, pp. 1941-1945, 2019.

[15] C. A. Balanis, Antenna Theory: Analysis and Design, Harper \& Row, Manhattan, NY, USA, 3rd edition, 1982.

[16] M. Barbuto, F. Trotta, F. Bilotti, and A. Toscano, "Circular polarized patch antenna generating orbital angular momentum," Progress In Electromagnetics Research, vol. 148, pp. 23-30, 2014.

[17] M. Zhu, X. Liang, Y. Yao et al., "Realization of various OAM divergence angles based on fabry-perot antenna design," in Proceedings of the 2017 Sixth Asia-Pacific Conference On Antennas And Propagation (APCAP), pp. 1-2, Xi'an, China, October 2017.

[18] N. R. Labadie, S. K. Sharma, and G. M. Rebeiz, "A circularly polarized multiple radiating mode microstrip antenna for satellite receive applications," IEEE Transactions on Antennas and Propagation, vol. 62, no. 7, pp. 3490-3500, 2014.

[19] M. Barbuto, M.-A. Miri, A. Alù, F. Bilotti, and A. Toscano, "A topological design tool for the synthesis of antenna radiation patterns," IEEE Transactions on Antennas and Propagation, vol. 68 , no. 3, pp. 1851-1859, 2020

[20] Z. Zhang, S. Xiao, Y. Li, and B.-Z. Wang, "A circularly polarized multimode patch antenna for the generation of multiple orbital angular momentum modes," IEEE Antennas and Wireless Propagation Letters, vol. 16, pp. 521-524, 2017.

[21] Y. Yao, X. Liang, R. Jin, and J. Geng, "Analysis of focusing orbital angular momentum wave using fabry-perot cavity," Journal of Communications and Information Networks, vol. 4, no. 3, pp. 9-17, 2019. 